\section{P100 EXPLORING WHY A COMPLEX INTERVENTION PILOTED IN GENERAL PRACTICES DID NOT RESULT IN AN INCREASE IN CHLAMYDIA SCREENING AND DIAGNOSIS: A QUALITATIVE EVALUATION USING THE FIDELITY OF IMPLEMENTATION MODEL}

${ }^{1}$ Rosalie Allison, ${ }^{1}$ Donna Lecky, ${ }^{2}$ Katy Town, ${ }^{1}$ Claire Rugman, ${ }^{1}$ Ellie Ricketts, ${ }^{1}$ Nina Ockendon-Powell, ${ }^{2}$ Kate Folkard, ${ }^{2}$ Kevin Dunbar, ${ }^{1}$ Cliodna McNulty*. ${ }^{1}$ Primary Care Unit, Public Health England, Gloucester, UK; ${ }^{2}$ National Chlamydia Programme, Public Health England, London, UK

10.1136/sextrans-2016-052718.154

Background To facilitate opportunistic chlamydia screening in general practices, a complex intervention (3Cs and HIV), based on the previously successful CIRT trial, was implemented across England. The intervention, to encourage practice staff to routinely offer chlamydia testing, only increased chlamydia screening in larger practices or in those offered incentives.

Aims a) Explore why the modified intervention did not increase screening across all general practices. b) Suggest recommendations for future intervention implementation.

Methods Phone interviews were carried out with 26 GP staff exploring their opinions on the workshops and intervention implementation in practice. Interview transcripts were thematically analysed and further examined using the fidelity of implementation model.

Results Participants were positive about the workshops but attendee numbers were low. Often, the intervention content was not adhered to: practice staff were unaware of any on-going trainer support; computer prompts were only added to the female contraception template; patients were not encouraged to complete the test immediately; and videos and posters were not utilised, as suggested. Staff reported that financial incentives, themselves, were not a motivator; competing priorities and time were identified as major barriers.

Conclusions Not adhering to the exact intervention model may explain the lack of significant increases in chlamydia screening. To increase fidelity of implementation and consequently improve likelihood of increased screening, the intervention needs to have: more specific action planning; computer prompts added to systems and used; all staff attend the workshop; and on-going practice staff support.

\section{P101 LATE PRESENTATION OF HIV (HUMAN IMMUNODEFICIENCY VIRUS) INFECTION AT A DISTRICT GENERAL HOSPITAL}

Ceri Williams*, Soumeya Cherif, Susannah Danino, Kathir Yoganathan. Singleton Hospital, Swansea, Wales, UK

\subsection{6/sextrans-2016-052718.155}

Background/introduction A significant proportion of patients (390/6360 (6.1\%) in 2012 nationally) present with an AIDS defining illness yearly despite increasing awareness and recognition of HIV. In 2012 the British HIV Association (BHIVA) suggested newly diagnosed patients should commence AntiRetroviral Therapy (ART) if their CD4 count $<350$ cells $/ \mathrm{mm}^{3}$, they have an AIDS defining illness or a neurological complication.

Aim(s)/objectives A re-audit was performed following previous audits in 2006/07 and 2011/2012 to ascertain whether late presentation has improved.
Methods A retrospective study compiled data from case notes of the newly diagnosed between 01/01/2014 and 31/12/2015. Defining late presenters as a CD4 count $<350$ cells $/ \mathrm{mm}^{3}$ or an AIDS defining illness.

Results 100 patients were identified, 33 were transfers and excluded. 67 remained of which $82.1 \%$ were male and $17.9 \%$ female. 52.2\% were late presenters and $25.4 \%$ had an AIDS defining illness of which 9 had PCP, 6 had oesophageal candidiasis, 1 had cryptococcal meningitis and 1 had OHL. Overall $35.8 \%$ had a CD4 <200 cells/ $/ \mathrm{mm}^{3}$ (42.9\% in 2011/2012 audit) and $68 \%$ of the late presenters.

Discussion/conclusion An improvement was identified in patients presenting with a CD4 count $<350$ cells $/ \mathrm{mm}^{3}$ (52.2\%) compared with 2011/12 audit (55.7\%). A high proportion continue to present with AIDS defining illnesses or depleted CD4 levels despite growing awareness of HIV and accessibility to health care. Poor prognosis, increasing morbidity and mortality is associated with late presentation. Atypical and opportunistic infections should prompt HIV testing amongst clinicians in both primary and secondary care along with improving patient education and contact tracing to minimise late presentation.

\section{P102 PRIMARY CARE SUPPORTED BY LEVEL 3 SEXUAL HEALTH CAN PROVIDE A HIGHLY ACCEPTABLE STI SERVICE TO MEN WHO HAVE SEX WITH MEN (MSM)}

${ }^{1}$ Tamuka Gonah*, ' ${ }^{1}$ odie Scrivener, ${ }^{1}$ Isata Gando, ${ }^{2,3}$ Daniel Richardson. ${ }^{1}$ Brighton Station Health Centre (careUK Ltd); ${ }^{2}$ Brighton \& Sussex University Hospitals NHS Trust, UK; ${ }^{3}$ Brighton \& Sussex Medical School

\subsection{6/sextrans-2016-052718.156}

Background/introduction Future health interventions for MSM (HPV vaccination \& HIV-PrEP) may need to be delivered in primary care: services need to be acceptable to MSM and aware of patient's sexuality. We are a walk in primary care and level 2 sexual health service in a city with a large MSM population supported by the local level 3 integrated sexual health service.

Aim(s)/objectives To measure the acceptability of an STI service in primary care.

Methods We offered an anonymous patient survey in the service between January and February 2016.

Results 93/120 (83\%) surveys were completed. 62/93 (67\%) of participants were male: 45/93 (48\%) identified as MSM. 91/93 (98\%) were satisfied with the clinical environment, 92/93 (99\%) said the service was accessible, 92/93 (99\%) would use the service again and $90 / 9 \%$ (97\%) would recommend the service to family and friends. 24/45 (53\%) MSM and 23/48 (48\%) nonMSM had previously used primary care for STI screening. 19/45 (42\%) MSM and 12/48 (25\%) non-MSM previously had Chlamydia. 27/45 (60\%) MSM, 7/48 (15\%) non-MSM previously had Gonorrhoea. 7/45 (16\%) MSM and 2/48 (4\%) of non-MSM were previously diagnosed with Syphilis. 5/45 (11\%) of MSM and $8 / 48(17 \%)$ of non-MSM said their GP was not aware of their sexuality. 9/45 (20\%) MSM and 7/38 (18\%) non-MSM voluntarily informed their GP of their sexuality. 15/45 (33\%) of MSM and 13/48 (27\%) of non-MSM were asked about their sexuality by their GP.

Discussion/conclusion Our primary care STI service is acceptable to patients who appear to attend primary care for sexual health including MSM with high rates of STIs. Systems to determine sexuality in primary care will be necessary for implementation of HPV vaccination and other health interventions in MSM 